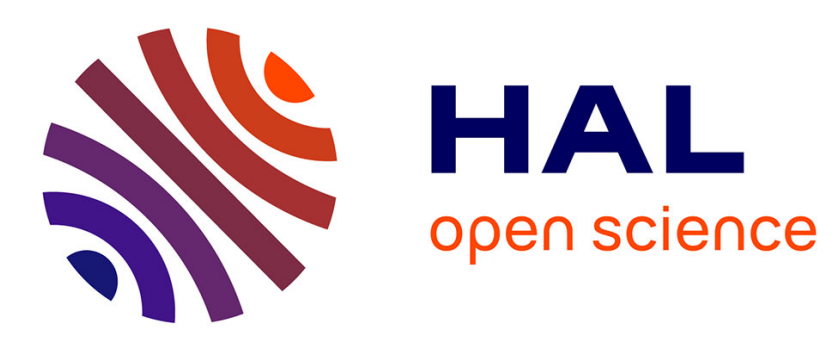

\title{
BSDE representations for optimal switching problems with controlled volatility
}

Romuald Elie, Idris Kharroubi

\section{To cite this version:}

Romuald Elie, Idris Kharroubi. BSDE representations for optimal switching problems with controlled volatility. Stochastics and Dynamics, 2014, 14, pp.1450003. 10.1142/S0219493714500038 . hal01103699

\section{HAL Id: hal-01103699 \\ https://hal.science/hal-01103699}

Submitted on 15 Jan 2015

HAL is a multi-disciplinary open access archive for the deposit and dissemination of scientific research documents, whether they are published or not. The documents may come from teaching and research institutions in France or abroad, or from public or private research centers.
L'archive ouverte pluridisciplinaire HAL, est destinée au dépôt et à la diffusion de documents scientifiques de niveau recherche, publiés ou non, émanant des établissements d'enseignement et de recherche français ou étrangers, des laboratoires publics ou privés. 


\title{
BSDE representations for optimal switching problems with controlled volatility
}

\author{
Romuald ELIE \& Idris KHARROUBI \\ CEREMADE, CNRS, UMR 7534, \\ Université Paris-Dauphine, \\ and CREST \\ \{elie,kharroubi\}@ceremade.dauphine.fr
}

\begin{abstract}
This paper provides two different strong BSDE representations for optimal switching problems in the case where the dynamics of the underlying diffusion process depends on the current value of the switching mode. These new representations are valid in a non-Markovian framework and make use of either one-dimensional constrained BSDEs with jumps or multidimensional BSDEs with oblique reflections, thus extending the framework considered by $\mathrm{Hu}$ and Tang [12. In particular, the numerical resolution of the corresponding switching problem can therefore be treated via the entirely probabilistic schemes presented in [4] or 8].
\end{abstract}

Key words: Stochastic control, Switching problems, Reflected BSDE.

MSC Classification (2000): 93E20, 60H30, 60J75.

\section{Introduction}

This paper is dedicated to the obtention of a probabilistic representation for a general form of continuous optimal switching problems. Consider for example an electricity producer trying to maximize its production rentability by switching between $m$ possible modes of production based on different commodities. Since the agent is a large investor on the market, we suppose that the dynamics of the considered commodities are modified by its current mode of production. Hence, the commodities have the following dynamics:

$$
X_{t}^{\alpha}=X_{0}+\int_{0}^{t} b_{\alpha_{s}}\left(s, X_{s}^{\alpha}\right) d s+\int_{0}^{t} \sigma_{\alpha_{s}}\left(s, X_{s}^{\alpha}\right) d W_{s}, \quad 0 \leq t \leq T,
$$

where $\alpha$ is a switching control process valued in $\mathcal{I}:=\{1, \ldots, m\}$. Each mode of production has its own profit design and the agent needs to solve the following switching control 
problem

$$
J_{0}^{*}:=\sup _{\alpha} \mathbf{E}\left[g_{\alpha_{T}}\left(X_{T}^{\alpha}\right)+\int_{0}^{T} \psi_{\alpha_{s}}\left(s, X_{s}^{\alpha}\right) d s+\sum_{0<\tau_{k} \leq T} c_{\alpha_{\tau_{k}^{-}}, \alpha_{\tau_{k}}}\right],
$$

where $\left(\tau_{k}\right)_{k}$ denotes the chosen jump times of the control $\alpha$. Any switch is penalized by a cost given by the function $c$. Of course, one of the mode of production may possibly consist in buying electricity directly on a financial market. More details on the practical implications of this type of optimal switching problem are given in [2] or [13].

Whenever the mode of production $\alpha$ does not influence the dynamics of the underlying $X$, 6] provides a probabilistic representation of the value process in terms of multidimensional reflected BSDE. Their approach extends the results of [10] obtained in the particular case where $m=2$. Allowing the drift of $X$ to depend on $\alpha$ and imposing the invertibility of its volatility, $\mathrm{Hu}$ and Tang [12] obtain a weak BSDE representation of $J_{0}^{*}$. Nevertheless, they only consider cases where the switching strategy barely affects the dynamics of the underlying diffusion, since the volatility of the commodities always remains unchanged. One may naturally wonder if such a probabilistic representation occurs in a framework where the volatility function $\sigma$ depends on the current switching mode $\alpha_{t}$ at time $t$. This paper brings a positive answer to this question and identifies $J_{0}^{*}$ as the value at time 0 of the strong solution of a multidimensional BSDE with oblique reflections. It is worth noticing that the solution of the corresponding BSDE can be approximated numerically using the probabilistic scheme studied in [4].

As observed recently in [9], the solution of multidimensional reflected BSDEs considered in 12] rewrites also in terms of the minimal solution of a one-dimensional constrained BSDE with jumps. The idea consists in letting the strategy $\alpha$ jump randomly between the different modes of production and relies on the reinterpretation of multidimensional switching reflections in terms of constraints on the jump component of the new BSDE. This kind of correspondence has first been observed by Bouchard [1] via PDE arguments and we show that it remains valid in the framework considered here. Hence, $J_{0}^{*}$ also rewrites as the value at time 0 of the minimal solution of a well chosen one-dimensional constrained BSDE with jumps. This type of BSDE may also be solved numerically via the probabilistic scheme presented in [8].

The rest of the paper is organized as follows. The next section introduces properly the optimal switching problem of interest. The third section recalls the weak representation of $\mathrm{Hu}$ and Tang [12] and presents our alternative strong representations via multidimensional reflected BSDEs or constrained BSDEs with jumps. The last section is dedicated to the proofs and details the introduction of a well chosen family of multidimensional reflected BSDE, which allows to represent the optimal switching strategy associated to the problem. Since all our arguments are based on purely probabilistic tools, we choose to present them in a possibly non-Markovian framework. 
Notations. Throughout this paper, we are given a finite terminal time $T$ and a probability space $(\Omega, \mathcal{G}, \mathbf{P})$ endowed with a $d$-dimensional standard Brownian motion $W=\left(W_{t}\right)_{t \geq 0}$ and an independent Poisson random measure $\mu$ on $\mathbb{R}_{+} \times \mathcal{I}$ of the form $\mu=\sum_{i \geq 1} \delta_{\tau_{i}, \zeta_{i}}$, where $\mathcal{I}:=\{1, \ldots, m\}$, with intensity measure $\lambda(d i) d t$ for some finite measure $\lambda$ on $\mathcal{I}$ with $\lambda(i)$ $>0$ for all $i \in \mathcal{I}$. $\sigma(\mathcal{I})$ denotes the $\sigma$-algebra of subsets of $\mathcal{I}$. For $x=\left(x_{1}, \ldots, x_{\ell}\right) \in$ $\mathbb{R}^{\ell}$ with $\ell \in \mathbb{N}$, we set $|x|=\sqrt{\left|x_{1}\right|^{2}+\cdots+\left|x_{\ell}\right|^{2}}$ the Euclidean norm. For $x, y \in \mathbb{R}^{\ell}$ with $\ell \in \mathbb{N},\langle x, y\rangle$ stands for the euclidean scalar product. We denote by $\mathbb{G}=\left(\mathcal{G}_{t}\right)_{t \geq 0}$ (resp. $\mathbb{F}$ $\left.=\left(\mathcal{F}_{t}\right)_{t \geq 0}\right)$ the augmentation of the natural filtration generated by $W$ and $\mu$ (resp. by $W$ ), and by $\mathcal{P}_{\mathbb{G}}\left(\right.$ resp. $\mathcal{P}_{\mathbb{F}}, \mathcal{P} \mathcal{M}(\mathbb{G}), \mathcal{P} \mathcal{M}(\mathbb{F})$ ) the $\sigma$-algebra of $\mathbb{G}$-predictable (resp. $\mathbb{F}$-predictable $\mathbb{G}$-progressive, $\mathbb{F}$-progressive) subsets of $\Omega \times[0, T]$. We denote by $\mathcal{S}_{\mathbb{G}}^{2}$ (resp. $\mathcal{S}_{\mathbb{F}}^{\mathbf{2}}$ ) the set of real-valued càd-làg $\mathbb{G}$-adapted (resp. continuous $\mathbb{F}$-adapted) processes $Y=\left(Y_{t}\right)_{0 \leq t \leq T}$ such that

$$
\|Y\|_{\mathcal{S}^{2}}:=\left(\mathbf{E}\left[\sup _{0 \leq t \leq T}\left|Y_{t}\right|^{2}\right]\right)^{\frac{1}{2}}<\infty .
$$

$\mathbf{L}^{2}(\mathbf{0}, \mathbf{T})$ is the set of real-valued processes $\phi=\left(\phi_{t}\right)_{0 \leq t \leq T}$ such that

$$
\|\phi\|_{\mathbf{L}^{2}(\mathbf{0}, \mathbf{T})}:=\left(\mathbf{E}\left[\int_{0}^{T}\left|\phi_{t}\right|^{2} d t\right]\right)^{\frac{1}{2}}<\infty
$$

and $\mathbf{L}_{\mathbb{F}}^{2}(\mathbf{0}, \mathbf{T})$ (resp. $\left.\mathbf{L}_{\mathbb{G}}^{2}(\mathbf{0}, \mathbf{T})\right)$ is the subset of $\mathbf{L}^{\mathbf{2}}(\mathbf{0}, \mathbf{T})$ consisting of $\mathcal{P} \mathcal{M}(\mathbb{F})$-measurable (resp. $\mathcal{P} \mathcal{M}(\mathbb{G})$-measurable) processes. $\mathbf{L}_{\mathbb{F}}^{2}(\mathbf{W})$ (resp. $\left.\mathbf{L}_{\mathbb{G}}^{2}(\mathbf{W})\right)$ is the set of $\mathbb{R}^{d}$-valued $\mathcal{P}_{\mathbb{F}^{-}}$ measurable (resp. $\mathcal{P}_{\mathbb{G}}$-measurable) processes $Z=\left(Z_{t}\right)_{0 \leq t \leq T} \in \mathbf{L}_{\mathbb{F}}^{2}(\mathbf{0}, \mathbf{T})$ (resp. $\mathbf{L}_{\mathbb{G}}^{2}(\mathbf{0}, \mathbf{T})$ ) . $\mathbf{L}^{2}(\mu)$ is the set of $\mathcal{P} \otimes \sigma(\mathcal{I})$-measurable maps $U: \Omega \times[0, T] \times \mathcal{I} \rightarrow \mathbb{R}$ such that

$$
\|U\|_{\mathbf{L}^{2}(\mu)}:=\left(\mathbf{E}\left[\int_{0}^{T} \int_{\mathcal{I}}\left|U_{t}(i)\right|^{2} \lambda(d i) d t\right]\right)^{\frac{1}{2}}<\infty .
$$

$\mathbf{A}_{\mathbb{F}}^{2}$ (resp. $\left.\mathbf{A}_{\mathbb{G}}^{2}\right)$ is the closed subset of $\mathcal{S}_{\mathbb{F}}^{2}\left(\right.$ resp. $\left.\mathcal{S}_{\mathbb{G}}^{2}\right)$ consisting of nondecreasing processes $K=\left(K_{t}\right)_{0 \leq t \leq T}$ with $K_{0}=0$. Finaly, for $t \in[0, T], \mathcal{T}_{t}$ denotes the set of $\mathbb{F}$-stopping times $\tau$ such that $\tau \in[t, T], \mathbf{P}$-a.s.. For ease of notation, we omit in all the paper the dependence in $\omega \in \Omega$, whenever it is not relevant.

\section{The general optimal switching problem}

Given the set $\mathcal{I}=\{1, \ldots, m\}$ and the maturity $T$, a switching strategy $\alpha$ consists in a sequence $\alpha:=\left(\tau_{k}, \zeta_{k}\right)_{k \geq 1}$, where $\left(\tau_{k}\right)_{k \geq 1}$ is an increasing sequence of $\mathbb{F}$-stoppping times smaller than $T$, and $\zeta_{i}$ are $\mathcal{F}_{\tau_{i}}$-measurable random variables valued in $\mathcal{I}$. To a strategy $\alpha=\left(\tau_{k}, \zeta_{k}\right)_{k \geq 1}$ and an initial regime $i_{0}$, we naturally associate the state process $\left(\alpha_{t}\right)_{t \leq T}$ defined by

$$
\alpha_{t}:=\sum_{k \geq 0} \zeta_{k} \mathbf{1}_{\left[\tau_{k}, \tau_{k+1}\right)}(t), \quad 0 \leq t \leq T,
$$

with $\tau_{0}=0$ and $\zeta_{0}=i_{0}$. We denote by $\mathcal{A}$ the set of admissible strategies and $\mathcal{A}_{i}$ the subset of strategies starting from state $i \in \mathcal{I}$ at time 0 :

$$
\mathcal{A}_{i}:=\left\{\alpha \in \mathcal{A}: \alpha_{0}=i\right\} .
$$


Given a strategy $\alpha \in \mathcal{A}$ and an initial condition $X_{0} \in \mathbb{R}^{d}$, we define the controlled process $X^{\alpha}$ by

$$
X_{t}^{\alpha}=X_{0}+\int_{0}^{t} b_{\alpha_{s}}\left(s, X_{s}^{\alpha}\right) d s+\int_{0}^{t} \sigma_{\alpha_{s}}\left(s, X_{s}^{\alpha}\right) d W_{s}, \quad 0 \leq t \leq T
$$

where the functions $b_{i}$ and $\sigma_{i}$ are $\mathcal{P} \mathcal{M}(\mathbb{F}) \otimes \mathcal{B}\left(\mathbb{R}^{d}\right)$-measurable, for any $i \in \mathcal{I}$. In order to ensure the existence of such a controlled process, we impose the following assumption:

(H1)

(i) $b(., 0)$ and $\sigma(., 0)$ are square integrable:

$$
\mathbb{E} \int_{0}^{T}\left(\left|b_{i}(t, 0)\right|^{2}+\left|\sigma_{i}(t, 0)\right|^{2}\right) d t<\infty, \quad i \in \mathcal{I} .
$$

(ii) $b$ and $\sigma$ are Lipschitz continuous: there exists a constant $L$ such that

$$
\left|b_{i}(\omega, t, x)-b_{i}\left(\omega, t, x^{\prime}\right)\right|+\left|\sigma_{i}(\omega, t, x)-\sigma_{i}\left(\omega, t, x^{\prime}\right)\right| \leq L\left|x-x^{\prime}\right|, \quad i \in \mathcal{I},
$$

for all $\left(t, x, x^{\prime}\right) \in[0, T] \times\left[\mathbb{R}^{d}\right]^{2}, \mathbf{P}-$ a.s. $\omega \in \Omega$.

Given a switching strategy $\alpha \in \mathcal{A}$ and the associated controlled process $X^{\alpha}$, we consider the total profit at horizon $T$ defined by

$$
J(\alpha):=\mathbf{E}\left[g_{\alpha_{T}}\left(X_{T}^{\alpha}\right)+\int_{0}^{T} \psi_{\alpha_{s}}\left(s, X_{s}^{\alpha}\right) d s-\sum_{0<\tau_{k} \leq T} c_{\zeta_{k-1}, \zeta_{k}}\left(\tau_{k}\right)\right],
$$

where the functions $g_{i}\left(\right.$ resp. $\left.\psi_{i}\right)$ are $\mathcal{F}_{T} \otimes \mathcal{B}\left(\mathbb{R}^{d}\right)\left(\right.$ resp. $\left.\mathcal{P} \mathcal{M}(\mathbb{F}) \otimes \mathcal{B}\left(\mathbb{R}^{d}\right)\right)$-measurable for all $i \in \mathcal{I}$ and $c_{i, j}$ are $\mathcal{P} \mathcal{M}(\mathbb{F})$-measurable for all $i, j \in \mathcal{I}$. As discussed in [2], this type of stochastic control problem is typically encountered by an agent maximizing the production

rentability of a given good by switching between $m$ possible modes of production based on different commodities.

We impose the following assumption which in particular ensures the proper definition of the expectation $J(\alpha)$ for all $\alpha \in \mathcal{A}$ :

(H2)

(i) The functions $|g|$ and $|\psi|$ are uniformly upper-bounded by the constants $\bar{g}$ and $\bar{\psi}$.

(ii) The cost function $c$ is lower-bounded, i.e. there exists a constant $\bar{c}>0$ such that

$$
c_{i, j}(\omega, t) \geq \bar{c}, \quad i, j \in \mathcal{I}, i \neq j, t \in[0, T], \mathbf{P}-\text { a.s. } \omega \in \Omega .
$$

Furthermore $c_{i, j} \in \mathcal{S}_{\mathbb{F}}^{2}$ for all $i, j \in \mathcal{I}$, and we have

$$
\inf _{t \in[0, T]}\left\{c_{i, j}(\omega, t)+c_{j, l}(\omega, t)-c_{i, l}(\omega, t)\right\} \geq \bar{c}, \quad i, j, l \in \mathcal{I}, j \neq i, j \neq l, \mathbf{P}-a . s . \omega \in \Omega .
$$


(iii) The terminal condition $g$ satisfies the following structural condition:

$$
g_{i}(\omega, x) \geq \max _{j \in \mathcal{I}}\left\{g_{j}(\omega, x)-c_{i, j}(\omega, T)\right\}, \quad x \in \mathbb{R}^{d}, i \in \mathcal{I}, \mathbf{P}-\text { a.s. } \omega \in \Omega .
$$

Remark 2.1. Assumption (H2) simply provides the classical framework for the study of optimal switching problems. (i)-(ii) ensures the well-posedness of the problem, (ii) makes indirect switching strategy irrelevant and (iii) ensures the non-optimality of a switching at maturity.

Given the starting initial production mode $i_{0}$, solving the switching problem consists in finding a strategy $\alpha^{*} \in \mathcal{A}_{i_{0}}$ such that

$$
J\left(\alpha^{*}\right)=J_{i_{0}}^{*}:=\sup _{\alpha \in \mathcal{A}_{i_{0}}} J(\alpha) .
$$

Such a strategy $\alpha^{*}$ is called optimal.

We first observe that the optimal switching problem over $\mathcal{A}$ can be restricted to the consideration of finite strategies $\mathcal{D}:=\cup_{i \in \mathcal{I}} \mathcal{D}_{i}$, with

$$
\mathcal{D}_{i}:=\left\{\alpha=\left(\tau_{k}, \zeta_{k}\right)_{k \geq 1} \in \mathcal{A}_{i} \mid \mathbf{P}\left(\tau_{k}<T, \quad \forall k \geq 1\right)=0\right\}, \quad i \in \mathcal{I} .
$$

Proposition 2.1. Under (H1) and (H2), the supremum of $J$ over $\mathcal{A}_{i}$ coincides with the one of $J$ over $\mathcal{D}_{i}$, that is

$$
\sup _{\alpha \in \mathcal{A}_{i}} J(\alpha)=\sup _{\alpha \in \mathcal{D}_{i}} J(\alpha), \quad i \in \mathcal{I}
$$

Proof. Fix $i \in \mathcal{I}$ and consider a strategy $\alpha=\left(\tau_{k}, \zeta_{k}\right)_{k \geq 0} \in \mathcal{A}_{i}$. Suppose that $\alpha \notin \mathcal{D}_{i}$ and introduce $B:=\left\{\omega \in \Omega \mid \tau_{n}(\omega)<T, \forall n \geq 1\right\}$ so that $\mathbf{P}(B)>0$. We derive from (H2) (i) and (ii) that

$$
J(\alpha) \leq \bar{g}+T \bar{\psi}-\mathbf{E}\left[\mathbf{1}_{B} \sum_{0<\tau_{k} \leq T} \bar{c}\right]=-\infty,
$$

and directly deduce (2.4).

\section{Optimal switching and BSDEs}

In this section, we provide several BSDE representations for the solution of the switching problem (2.3). We first recall the weak representation obtained by $\mathrm{Hu}$ and Tang [12] under strong restrictions on the dependence of the diffusion coefficients $(b, \sigma)$ with respect to the control $\alpha$. Getting rid of these restrictions, we introduce two new strong probabilistic representations via either multidimensional reflected BSDEs or constrained BSDEs with jumps. 


\subsection{Weak BSDE representation}

In the particular case where the volatility coefficient $\sigma$ is not controlled, $\mathrm{Hu}$ and Tang [12] relate the solution $J_{i_{0}}^{*}, i_{0} \in \mathcal{I}$, of the optimal switching problem $(2.3)$ with a multidimensional BSDE with oblique reflections. More precisely, they work under the following assumption:

(H3)

(i) The functions $\sigma_{i}$ do not depend on $i \in \mathcal{I}$ and are invertible with bounded inverse.

(ii) The functions $\mu_{i}:=\sigma_{i}^{-1} b_{i}$ are bounded and Lipschitz continuous: there exists a constant $L$ such that

$$
\left|\mu_{i}(\omega, t, x)-\mu_{i}\left(\omega, t, x^{\prime}\right)\right| \leq L\left|x-x^{\prime}\right|, \quad\left(i, t, x, x^{\prime}\right) \in \mathcal{I} \times[0, T] \times\left[\mathbb{R}^{d}\right]^{2}, \quad \mathbf{P}-\text { a.s. } \omega \in \Omega .
$$

(iii) The cost functions $\left(c_{i, j}\right)$ are constant, for $i, j \in \mathcal{I}^{2}$.

They consider the following BSDE with oblique reflections

$$
\left\{\begin{array}{l}
\left(Y^{i}, Z^{i}, K^{i}\right)_{i \in \mathcal{I}} \in\left(\mathcal{S}_{\mathbb{F}}^{2} \times \mathbf{L}_{\mathbb{F}}^{\mathbf{2}}(\mathbf{W}) \times \mathbf{A}_{\mathbb{F}}^{\mathbf{2}}\right)^{m}, \\
Y_{t}^{i}=g_{i}\left(X_{T}\right)+\int_{t}^{T}\left(\psi_{i}\left(s, X_{s}\right)-\left\langle\mu_{i}\left(s, X_{s}\right), Z_{s}^{i}\right\rangle\right) d s-\int_{t}^{T}\left\langle Z_{s}^{i}, d W_{s}\right\rangle+K_{T}^{i}-K_{t}^{i}, \\
Y_{t}^{i} \geq \max _{j \in \mathcal{I}}\left\{Y_{t}^{j}-c_{i, j}(t)\right\}, \quad 0 \leq t \leq T, \\
\int_{0}^{T}\left[Y_{t}^{i}-\max _{j \in \mathcal{I}}\left\{Y_{t}^{j}-c_{i, j}(t)\right\}\right] d K_{t}^{i}=0, \quad i \in \mathcal{I},
\end{array}\right.
$$

where $X$ is the diffusion defined by

$$
X_{t}=X_{0}+\int_{0}^{t} \sigma\left(s, X_{s}\right) d W_{s}, \quad \forall t \geq 0
$$

Using a Girsanov transform argument, Hu and Tang [12] provide in Theorem 4.1 the following link between the optimal values $\left(J_{i}^{*}\right)_{i \in \mathcal{I}}$ and the BSDE (3.5).

Theorem 3.1 (Hu and Tang 2010). Suppose that (H1)-(H2)-(H3) hold and fix $i_{0} \in \mathcal{I}$. There exists a weak solution $\left({ }^{*} \mathbf{P},{ }^{*} W,{ }^{*} X,{ }^{*} Y,{ }^{*} Z\right)$ to the decoupled FBSDE (3.6)-(3.5) such that

$$
J_{i_{0}}^{*}={ }^{*} Y_{0}^{i_{0}}
$$

Under fewer assumptions, we provide in the next paragraph a more general relation between optimal switching problems and BSDEs with oblique reflections.

\subsection{Representation via multidimensional reflected BSDE}

We consider now the more natural multidimensional reflected BSDE

$$
\left\{\begin{array}{l}
\left(Y^{i}, Z^{i}, K^{i}\right)_{i \in \mathcal{I}} \in\left(\mathcal{S}_{\mathbb{F}}^{2} \times \mathbf{L}_{\mathbb{F}}^{\mathbf{2}}(\mathbf{W}) \times \mathbf{A}_{\mathbb{F}}^{\mathbf{2}}\right)^{m} \\
Y_{t}^{i}=g_{i}\left(X_{T}^{i}\right)+\int_{t}^{T} \psi_{i}\left(s, X_{s}^{i}\right) d s-\int_{t}^{T}\left\langle Z_{s}^{i}, d W_{s}\right\rangle+K_{T}^{i}-K_{t}^{i}, \\
Y_{t}^{i} \geq \max _{j \in \mathcal{I}}\left\{Y_{t}^{j}-c_{i, j}(t)\right\}, \quad 0 \leq t \leq T \\
\int_{0}^{T}\left[Y_{t}^{i}-\max _{j \in \mathcal{I}}\left\{Y_{t}^{j}-c_{i, j}(t)\right\}\right] d K_{t}^{i}=0, \quad i \in \mathcal{I},
\end{array}\right.
$$


where $\left(X^{i}\right)_{i \in \mathcal{I}}$ is the diffusion process defined by

$$
X_{t}^{i}=X_{0}+\int_{0}^{t} b_{i}\left(s, X_{s}^{i}\right) d s+\int_{0}^{t} \sigma_{i}\left(s, X_{s}^{i}\right) d W_{s}, \quad t \geq 0, \quad i \in \mathcal{I} .
$$

Under (H1) and (H2), the existence of a unique solution to the BSDE (3.7) is ensured by Theorem 3.2 and Theorem 4.6 in [1] or Theorem 2.1 in [3]. The main result of the paper is the following.

Theorem 3.2. If (H1) and (H2) are in force, we have

$$
J_{i_{0}}^{*}=Y_{0}^{i_{0}}, \quad i_{0} \in \mathcal{I},
$$

where $(Y, Z)$ is the solution of the BSDE (3.7).

Section 4.2 of this paper is dedicated to the characterization of an optimal strategy $\alpha^{*} \in \mathcal{A}_{i_{0}}$ solving problem 2.3 via a well chosen family of multidimensional reflected BSDE, see Proposition 4.2. As detailed in Remark 4.1 below, the proof of Theorem 3.2 is a direct consequence of this more general dynamic representation.

\subsection{Representation via constrained BSDE with jumps}

An alternative to the consideration of multidimensional reflected BSDEs consists on the introduction of an independent random mode of production $\left(I_{t}\right)_{t \leq T}$ and the corresponding forward diffusion $X^{I}$ with switching regimes. Considering this two-dimensional transmutationdiffusion process $\left(I, X^{I}\right)$ as a new forward process, the introduction of a well chosen constrained BSDE with jumps allows for the representation of the solution to the optimal switching problem 2.3. This type of correspondence has already been observed in [9] when the dynamics of the forward process $X$ does not depend on the mode of production. We prove hereafter that it remains valid in the general framework considered here.

Consider the one-dimensional constrained BSDE with jumps:

$$
\left\{\begin{array}{l}
(\bar{Y}, \bar{Z}, \bar{U}, \bar{K}) \in \mathcal{S}_{\mathbb{G}}^{2} \times \mathbf{L}_{\mathbb{G}}^{2}(\mathbf{W}) \times \mathbf{L}^{2}(\mu) \times \mathbf{A}_{\mathbb{G}}^{2} \\
\bar{Y}_{t}=g_{I_{T}}\left(X_{T}^{I}\right)+\int_{t}^{T} \psi_{I_{s}}\left(s, X_{s}^{I}\right) d s+\bar{K}_{T}-\bar{K}_{t}-\int_{t}^{T}\left\langle\bar{Z}_{s}, d W_{s}\right\rangle-\int_{t}^{T} \int_{\mathcal{I}} \bar{U}_{s}(i) \mu(d s, d i) \\
\bar{U}_{t}(i) \leq c_{I_{t^{-}}, i}(t), \quad d \mathbf{P} \otimes d t \otimes \lambda(d i) \text { a.e. }
\end{array}\right.
$$

where the process $\left(I, X^{I}\right)$ is defined on $[0, T]$ as the unique solution of

$$
\left\{\begin{array}{l}
I_{t}=I_{0}+\int_{0}^{t} \int_{\mathcal{I}}\left(i-I_{t^{-}}\right) \mu(d t, d i) \\
X_{t}^{I}=X_{0}+\int_{0}^{t} b_{I_{s}}\left(s, X_{s}^{I}\right) d s+\int_{0}^{t} \sigma_{I_{s}}\left(s, X_{s}^{I}\right) d W s
\end{array}\right.
$$

We recall that the poisson measure $\mu$ is independent of the Brownian motion $W$. This constrained BSDE enters into the class of BSDEs considered recently in [9]. As detailed in the Section 4.3 hereafter, (H1)-(H2) ensures the existence of a minimal solution $(\bar{Y}, \bar{Z}, \bar{U}, \bar{K})$ to 3.9$)$ : for any other solution $\left(\bar{Y}^{\prime}, \bar{Z}^{\prime}, \bar{U}^{\prime}, \bar{K}^{\prime}\right)$ to 3.9 we have

$$
\bar{Y}_{t} \leq \bar{Y}_{t}^{\prime}, \quad t \in[0, T], \mathbf{P}-\text { a.s. }
$$


Adequately, this solution also provides a probabilistic representation for the solution $J_{i_{0}}^{*}$ of the switching problem 2.3 .

Theorem 3.3. If $(\boldsymbol{H} 1)$ and $(\boldsymbol{H} 2)$ are in force, there exists a unique minimal solution $(\bar{Y}, \bar{Z}, \bar{U}, \bar{K})$ to 3.9 and we have

$$
J_{I_{0}}^{*}=\bar{Y}_{0}
$$

The proof of Theorem 3.3 also requires the consideration of the similar family of multidimensional reflected BSDE and is reported in Section 4.3 .

Comparing to the existing literature, we notice that this result extends to a nonMarkovian framework the results obtained in [8] via PDE arguments.

\section{Optimal switching representation via a family of BSDEs}

This section is dedicated to the proofs of Theorem 3.2 and Theorem 3.3 and relies on a nice representation of the optimal switching strategy for problem 2.3 using a large family of reflected BSDE.

\subsection{The family of reflected BSDEs}

In the spirit of [5], we introduce the following family of reflected BSDE. Any element of the family will be characterized by a couple $(\nu, \eta)$ with $\nu$ an $\mathbb{F}$-stopping time valued in $[0, T]$ and $\eta$ an $\mathcal{F}_{\nu}$-measurable random variable taking values in $\mathbb{R}^{d}$. The set of such couple $(\nu, \eta)$ will be denoted $\mathcal{K}$.

For any parameter $(\nu, \eta) \in \mathcal{K}$, we consider the following reflected BSDE

$$
\left\{\begin{array}{l}
\left(Y^{\nu, i, \eta}, Z^{\nu, i, \eta}, K^{\nu, i, \eta}\right)_{i \in \mathcal{I}} \in\left(\mathcal{S}_{\mathbb{F}}^{2} \times \mathbf{L}_{\mathbb{F}}^{\mathbf{2}}(\mathbf{W}) \times \mathbf{A}_{\mathbb{F}}^{\mathbf{2}}\right)^{m} \\
Y_{t}^{\nu, i, \eta}=g_{i}\left(X_{T}^{\nu, i, \eta}\right)+\int_{t \wedge \nu}^{T} \psi_{i}\left(s, X_{s}^{\nu, i, \eta}\right) \mathbf{1}_{s \geq \nu} d s-\int_{t \wedge \nu}^{T}\left\langle Z_{s}^{\nu, i, \eta}, d W_{s}\right\rangle+K_{T}^{\nu, i, \eta}-K_{t}^{\nu, i, \eta} \\
Y_{t}^{\nu, i, \eta} \geq \max _{j \in \mathcal{I}}\left\{Y_{t}^{\nu, j, \eta}-c_{i, j}(t)\right\}, \quad 0 \leq t \leq T \\
\int_{0}^{T}\left[Y_{t}^{\nu, i, \eta}-\max _{j \in \mathcal{I}}\left\{Y_{t}^{\nu, j, \eta}-c_{i, j}(t)\right\}\right] d K_{t}^{\nu, i, \eta}=0
\end{array}\right.
$$

where $X^{\nu, i, \eta}$ is the diffusion defined by

$$
X_{t}^{\nu, i, \eta}=\eta \mathbf{1}_{t \geq \nu}+\int_{\nu}^{t} b_{i}\left(s, X_{s}^{\nu, i, \eta}\right) d s+\int_{\nu}^{t} \sigma_{i}\left(s, X_{s}^{\nu, i, \eta}\right) d W_{s}, \quad \forall t \geq 0 .
$$

Under (H1) and (H2), Theorem 4.2 in [11] provides the existence of a unique solution to 4.1), for any parameter $(\nu, \eta) \in \mathcal{K}$, and we denote by $\mathcal{O}^{\nu, ., \eta}$ the corresponding frontier for the domain of $Y^{\nu, ., \eta}$ defined by

$$
\mathcal{O}_{t}^{\nu, i, \eta}:=\max _{j \in \mathcal{I}}\left\{Y_{t}^{\nu, j, \eta}-c_{i, j}(t)\right\}, \quad i \in \mathcal{I}, \quad t \leq T .
$$

We aim at relating the solutions of this family of reflected BSDEs to an optimal strategy for the switching problem (2.3). The next proposition provides a stability property, a Snell

envelope representation and a global estimate on the family of processes $\left(Y^{\nu,,, \eta}\right)_{(\nu, \eta) \in \mathcal{K}}$. 
Proposition 4.1. If (H1) and (H2) are in force, the following holds.

(i) For any $\eta, \nu$ and $\nu^{\prime}$ such that $(\eta, \nu) \in \mathcal{K}, \nu$ an $\mathbb{F}$-stopping time such that $\nu \leq \nu^{\prime}$, we have $\left(X_{\nu^{\prime}}^{\nu, i, \eta}, \nu^{\prime}\right) \in \mathcal{K}$ and

$$
Y_{t}^{\nu, i, \eta}=Y_{t}^{\nu^{\prime}, i, X_{\nu^{\prime}}^{\nu, i, \eta}}, \quad \mathbf{P}-\text { a.s. }, \quad t \geq \nu^{\prime}, \quad i \in \mathcal{I} .
$$

(ii) For all $i \in \mathcal{I},(\eta, \nu) \in \mathcal{K}$ and $t \geq \nu$, we have the following representation

$$
Y_{t}^{\nu, i, \eta}=\underset{\tau \in \mathcal{T}_{t}}{\operatorname{ess} \sup } \mathbf{E}\left[\int_{t \wedge \nu}^{\tau} \psi_{i}\left(s, X_{s}^{\nu, i, \eta}\right) d s+\mathcal{O}_{\tau}^{\nu, i, \eta} \mathbf{1}_{\tau<T}+g_{i}\left(X_{T}^{\nu, i, \eta}\right) \mathbf{1}_{\tau=T} \mid \mathcal{F}_{t}\right] .
$$

(iii) There exists a constant $\bar{y}$ such that

$$
\sup _{(t, i, \nu, \eta) \in[0, T] \times \mathcal{I} \times \mathcal{K}}\left|Y_{t}^{\nu, i, \eta}\right| \leq \bar{y}, \quad \mathbf{P}-\text { a.s. }
$$

Proof. We prove each assertion separately.

(i) Fix $i \in \mathcal{I}$ and $\eta, \nu, \nu^{\prime}$ as required. Notice first that $X^{\nu, i, \eta}$ and $X^{\nu^{\prime}, i, X_{\nu^{\prime}}^{\nu, i, \eta}}$ solve the same SDE on $\left[\nu^{\prime}, T\right]$, namely

$$
X_{\nu^{\prime}}=X_{\nu^{\prime}}^{\nu, i, \eta} \quad \text { and } \quad d X_{t}=b_{i}\left(t, X_{t}\right) d t+\sigma_{i}\left(t, X_{t}\right) d W_{t}, \quad \text { for } t \geq \nu^{\prime} .
$$

Under (H1), equation 4.6 admits a unique solution and we have $X^{\nu^{\prime}, i, X_{\nu^{\prime}}^{\nu, i, \eta}}=X^{\nu, i, \eta}$ on $\left[\nu^{\prime}, T\right]$. We deduce that $\left(Y^{\nu^{\prime}, i, X_{\nu^{\prime}}^{\nu, i, \eta}}, Z^{\nu^{\prime}, i, X_{\nu^{\prime}}^{\nu, i, \eta}}, K^{\nu^{\prime}, i, X_{\nu^{\prime}}^{\nu, i, \eta}}\right)_{i \in \mathcal{I}}$ satisfies the same BSDE as $\left(Y^{\nu, i, \eta}, Z^{\nu, i, \eta}, K^{\nu, i, \eta}\right)_{i \in \mathcal{I}}$ on $\left[\nu^{\prime}, T\right]$. Under (H2), Theorem 4.2 in [11] provides uniqueness of solution to this BSDE and concludes the argumentation.

(ii) Fix $i \in \mathcal{I}$ and $(\eta, \nu) \in \mathcal{K}$. Regarding of 4.1), $\left(Y^{\nu, i, \eta}, Z^{\nu, i, \eta}, K^{\nu, i, \eta}\right)$ interprets as the solution of a one-dimensional reflected BSDE with single barrier $\mathcal{O}^{\nu, i, \eta}$. We deduce from Proposition 2.3 in [7] that $Y^{\nu, i, \eta}$ admits the Snell envelope representation (4.4).

(iii) Fix $(\nu, \eta) \in \mathcal{K}$. We know from equation (3.8) in the proof of Theorem 3.2 in [1] that $\left(Y^{\nu, ., \eta, n}, Z^{\nu, ., \eta, n}, K^{\nu, ., \eta, n}\right)_{n \in \mathbb{N}}$ converges in $\mathcal{S}_{\mathbb{F}}^{2} \times \mathbf{L}_{\mathbb{F}}^{2}(\mathbf{W}) \times \mathcal{S}_{\mathbb{F}}^{2}$ to $\left(Y^{\nu, ., \eta}, Z^{\nu, ., \eta}, K^{\nu, ., \eta}\right)$, where the sequence $\left(Y^{\nu, ., \eta, n}, Z^{\nu, ., \eta, n}, K^{\nu, ., \eta, n}\right)_{n \in \mathbb{N}}$ is defined recursively by

$Y_{t}^{\nu, i, \eta, 0}=g_{i}\left(X_{T}^{\nu, \eta}\right)+\int_{t \wedge \nu}^{T} \psi_{i}\left(s, X_{s}^{\nu, i, \eta}\right) d s-\int_{t \wedge \nu}^{T}\left\langle Z_{s}^{\nu, i, \eta, 0}, d W_{s}\right\rangle \quad$ and $\quad K_{t}^{\nu, i, \eta, 0}=0, i \in \mathcal{I}$,

and, for $n \geq 1$, by

$$
\left\{\begin{array}{l}
Y_{t}^{\nu, i, \eta, n}=g_{i}\left(X_{T}^{\nu, i, \eta}\right)+\int_{t \wedge \nu}^{T} \psi_{i}\left(s, X_{s}^{\nu, i, \zeta}\right) d s-\int_{t \wedge \nu}^{T}\left\langle Z_{t}^{\nu, i, \eta, n}, d W_{s}\right\rangle+K_{T}^{\nu, i, \eta, n}-K_{t}^{\nu, i, \eta, n}, \\
Y_{t}^{\nu, i, \eta, n} \geq \max _{j \in \mathcal{I}}\left\{Y_{t}^{\nu, j, \eta, n-1}-c_{i, j}(t)\right\}, \quad 0 \leq t \leq T, \\
\int_{0}^{T}\left[Y_{t}^{\nu, i, \eta, n}-\max _{j \in \mathcal{I}}\left\{Y_{t}^{\nu, j, \eta, n-1}-c_{i, j}(t)\right\}\right] d K_{t}^{\nu, i, \eta, n}=0, \quad i \in \mathcal{I} .
\end{array}\right.
$$

In order to derive (4.5), it thus suffices to prove by induction on $n$ that

$$
\left|Y_{t}^{\nu, i, \eta, n}\right| \leq(T-t+1) \max \{\bar{\psi}, \bar{g}\}, \quad \mathbf{P}-\text { a.s. }, \quad i \in \mathcal{I}, \quad 0 \leq t \leq T, \quad n \in \mathbb{N} .
$$


First, rewriting $Y^{\nu, ., \eta, 0}$ as a conditional expectation, we directly get

$$
\left|Y_{t}^{\nu, i, \eta, 0}\right| \leq(T-t) \bar{\psi}+\bar{g} \leq(T-t+1) \max \{\bar{\psi}, \bar{g}\}, \quad 0 \leq t \leq T, \quad i \in \mathcal{I} .
$$

Fix $n \in \mathbb{N}$ and suppose the result is true for $Y^{\nu, ., \eta, n}$. For $i \in \mathcal{I}$, using the representation of $Y^{\nu, i, \eta, n+1}$ as a Snell envelope given by Proposition 2.3 in [7], we derive

$$
Y_{t}^{\nu, i, \eta, n+1}=\operatorname{ess~sup}_{\tau \in \mathcal{T}_{t}} \mathbf{E}\left[\int_{t}^{\tau} \psi_{i}\left(s, X_{s}^{\nu, i, \eta}\right) \mathbf{1}_{s \geq \nu} d s+\mathcal{O}_{\tau}^{\nu, i, \eta, n+1} \mathbf{1}_{\tau<T}+g_{i}\left(X_{T}^{\nu, i, \eta}\right) \mathbf{1}_{\tau=T} \mid \mathcal{F}_{t}\right]
$$

where $\mathcal{O}_{s}^{\nu, i, \eta, n+1}:=\max _{j \in \mathcal{I}}\left\{Y_{s}^{\nu, j, \eta, n}-c_{i, j}(s)\right\}$, for $s \leq T$. Combining this representation with Assumption (H2)(i)-(ii) as well as the recursive estimate, we get

$$
\begin{aligned}
Y_{t}^{\nu, i, \eta, n+1} & \leq \underset{\tau \in \mathcal{T}_{t}}{\operatorname{esssup}} \mathbf{E}\left[(\tau-t) \bar{\psi}+(T-\tau+1) \max \{\bar{\psi}, \bar{g}\} \mathbf{1}_{\tau<T}+\bar{g} \mathbf{1}_{\tau=T} \mid \mathcal{F}_{t}\right] \\
& \leq(T-t+1) \max \{\bar{\psi}, \bar{g}\}, \quad 0 \leq t \leq T, \quad i \in \mathcal{I} .
\end{aligned}
$$

By induction and arbitrariness of $(\nu, \eta) \in \mathcal{K}$, we deduce 4.5.

\subsection{Relating the family to the optimal switching strategy}

We now prove the main result of this paper: the link between the optimal switching problem 2.3) and the initial value of the BSDE of type 4.1 with parameters $(\nu, \eta)=\left(0, X_{0}\right)$. This relation is obtained via the reinterpretation of an optimal switching strategy for problem 2.2 in terms of solutions to the family of multidimensional reflected BSDEs $\left(Y^{\nu, ., \eta}, Z^{\nu, ., \eta}, K^{\nu, ., \eta}\right)_{(\nu, \eta) \in \mathcal{K}}$ given by 4.1 .

For any $(\nu, \eta) \in \mathcal{K}$ and any $\mathcal{I}$-valued random variable $\zeta$, we naturally introduce the processes $Y^{\nu, \zeta, \eta}$ and $\mathcal{O}^{\nu, \zeta, \eta}$ defined by

$$
Y_{t}^{\nu, \zeta, \eta}:=\sum_{i \in \mathcal{I}} Y_{t}^{\nu, i, \eta} \mathbf{1}_{\zeta=i} \quad \text { and } \quad \mathcal{O}_{t}^{\nu, \zeta, \eta}:=\sum_{i \in \mathcal{I}} \mathcal{O}_{t}^{\nu, i, \eta} \mathbf{1}_{\zeta=i}
$$

Proposition 4.2. Let $\alpha^{*}=\left(\tau_{n}^{*}, \zeta_{n}^{*}\right)_{n \geq 0}$ be the strategy given by $\left(\tau_{0}^{*}, \zeta_{0}^{*}\right)=\left(0, i_{0}\right)$ with $i_{0} \in \mathcal{I}$ and defined recursively, for $n \geq 1$, by

$$
\begin{aligned}
& \tau_{n}^{*} \quad:=\quad \inf \left\{s \in\left[\tau_{n-1}^{*}, T\right] ; Y_{s}^{\tau_{n-1}^{*}, \zeta_{n-1}^{*}, X_{\tau_{n-1}^{*}}^{*}}=\mathcal{O}_{s}^{\tau_{n-1}^{*}, \zeta_{n-1}^{*}, X_{\tau_{n-1}^{*}}^{*}}\right\}, \\
& \zeta_{n}^{*} \quad \text { is s.t. } \quad \mathcal{O}_{\tau_{n}^{*}}^{\tau_{n-1}^{*}, \zeta_{n-1}^{*}, X_{\tau_{n-1}^{*}}^{*}}=Y_{\tau_{n}^{*}}^{\tau_{n}^{*}, \zeta_{n}^{*}, X_{\tau_{n}^{*}}^{*}}-c_{\zeta_{n-1}^{*}, \zeta_{n}^{*}}\left(\tau_{n}^{*}\right),
\end{aligned}
$$

with $X^{*}$ the diffusion defined by

$$
X_{t}^{*}=x_{0}+\sum_{n \geq 1} \int_{\tau_{n-1}^{*}}^{\tau_{n}^{*}} b_{\zeta_{n-1}^{*}}\left(s, X_{s}^{*}\right) \mathbf{1}_{s \leq t} d s+\sum_{n \geq 1} \int_{\tau_{n-1}^{*}}^{\tau_{n}^{*}} \sigma_{\zeta_{n-1}^{*}}\left(s, X_{s}^{*}\right) \mathbf{1}_{s \leq t} d W_{s}, \quad t \geq 0 .
$$

Under (H1)-(H2), the strategy $\alpha^{*}$ is optimal for the switching problem 2.2) and we have

$$
Y_{0}^{0, i_{0}, X_{0}}=J_{i_{0}}^{*}, \quad i_{0} \in \mathcal{I} .
$$


Remark 4.1. Notice that for $(\nu, \eta)=\left(0, X_{0}\right)$, the process $\left(Y^{\nu, i, \eta}, Z^{\nu, i, \eta}\right)_{i \in \mathcal{I}}$ simply interprets as the unique solution of the BSDE (3.7). Therefore, Theorem 3.2 is a direct corollary of Proposition 4.2 .

Proof. We fix $i_{0} \in \mathcal{I}$ and perform the proof in two steps.

Step 1. The strategy $\alpha^{*} \in \mathcal{D}_{i_{0}}$ and satisfies $Y_{0}^{0, i_{0}, X_{0}}=J\left(\alpha^{*}\right)$.

The representation (4.4) in Proposition 4.1 rewrites

$$
Y_{0}^{0, i_{0}, X_{0}}=\underset{\tau \in \mathcal{T}_{0}}{\operatorname{ess} \sup } \mathbf{E}\left[\int_{0}^{\tau} \psi_{i_{0}}\left(s, X_{s}^{0, i_{0}, x_{0}}\right) d s+\mathcal{O}_{\tau}^{0, i_{0}, X_{0}} \mathbf{1}_{\tau<T}+g_{i_{0}}\left(X_{T}^{0, i_{0}, x_{0}}\right) \mathbf{1}_{\tau=T}\right]
$$

Since the boundary $\mathcal{O}^{0, i_{0}, X_{0}}$ is continuous, the stopping time $\tau_{1}^{*}$ is optimal for 4.13 , see Proposition 2.3 in [7], and we get

$$
\begin{aligned}
Y_{0}^{0, i_{0}, X_{0}} & =\mathbf{E}\left[\int_{0}^{\tau_{1}^{*}} \psi_{i_{0}}\left(s, X_{s}^{0, i_{0}, x_{0}}\right) d s+\mathcal{O}_{\tau_{1}^{*}}^{0, i_{0}, X_{0}} \mathbf{1}_{\tau_{1}^{*}<T}+g_{i_{0}}\left(X_{T}^{0, i_{0}, x_{0}}\right) \mathbf{1}_{\tau_{1}^{*}=T}\right] \\
& =\mathbf{E}\left[\int_{0}^{\tau_{1}^{*}} \psi_{\zeta_{0}^{*}}\left(s, X_{s}^{*}\right) d s+\left(Y_{\tau_{1}^{*}}^{\tau_{1}^{*}, \zeta_{1}^{*}, X_{\tau_{1}^{*}}^{*}}-c_{\zeta_{0}^{*}, \zeta_{1}^{*}}\left(\tau_{1}^{*}\right)\right) \mathbf{1}_{\tau_{1}^{*}<T}+g_{\zeta_{0}^{*}}\left(X_{T}^{*}\right) \mathbf{1}_{\tau_{1}^{*}=T}\right],
\end{aligned}
$$

where the last equality follows from the definitions of $\zeta_{1}^{*}$ and $X^{*}$ as well as Part (i) of Proposition 4.1. When $\tau_{1}^{*}<T$, using the Snell envelope representation of $Y_{\tau_{1}^{*}}^{\tau_{1}^{*}, \zeta_{1}^{*}, X_{\tau_{1}^{*}}^{*}}$ given by (4.1), we deduce recursively that

$$
\begin{aligned}
Y_{0}^{0, i_{0}, X_{0}=} \mathbf{E}\left[\sum_{k=1}^{n} \int_{\tau_{k-1}^{*}}^{\tau_{k}^{*}} \psi_{\zeta_{k}^{*}}\left(s, X_{s}^{*}\right) d s+Y_{\tau_{n}^{*}}^{\tau_{n}^{*}, \zeta_{n}^{*}, X_{\tau_{n}^{*}}^{*}} \mathbf{1}_{\tau_{n}^{*}<T}\right. \\
\left.\quad-\sum_{k=1}^{n} c_{\zeta_{k-1}^{*}, \zeta_{k}^{*}}\left(\tau_{k}^{*}\right) \mathbf{1}_{\tau_{k}^{*}<T}+\sum_{k=1}^{n} g_{\zeta_{k-1}^{*}}\left(X_{T}^{*}\right) \mathbf{1}_{\tau_{k-1}^{*}<\tau_{k}^{*}=T}\right], \quad n \in \mathbb{N}^{*} .
\end{aligned}
$$

We now prove $\alpha^{*} \in \mathcal{D}_{i_{0}}$ and assume on the contrary that $p:=\mathbf{P}\left(\tau_{n}^{*}<T, \forall n \in \mathbb{N}\right)>0$. Combining (H2)(i)-(ii), 4.5) and 4.14, we derive

$$
\begin{aligned}
& Y_{0}^{0, i_{0}, x_{0}} \leq \bar{\psi} T+\mathbf{E}\left[\sup _{s \leq T}\left|Y_{s}^{\tau_{n}^{*}, \zeta_{n}^{*}, X_{\tau_{n}^{*}}^{*} \mid}\right|\right]-n \bar{c} \mathbf{P}\left(\tau_{k}^{*}<T, \forall k \geq 0\right)+\bar{g} \\
& \leq \bar{\psi} T+\bar{y}-n \bar{c} p+\bar{g}, \quad n \in \mathbb{N}^{*} \text {. }
\end{aligned}
$$

Sending $n$ to infinity in the previous expression leads to $Y_{0}^{0, i_{0}, X_{0}}=-\infty$ which contradicts $Y^{0, i_{0}, x_{0}} \in \mathcal{S}_{\mathbb{F}}^{2}$. Therefore $\mathbf{P}\left(\tau_{k}^{*}<T, \forall k \geq 0\right)=0$ and $\alpha^{*} \in \mathcal{D}_{i_{0}}$. Finally, taking the limit as $n \rightarrow \infty$ in 4.14 leads to 4.12 .

Step 2. The strategy $\alpha^{*}$ is optimal.

According to Proposition 2.1, it suffices to consider finite strategies and we pick any $\alpha=$ $\left(\tau_{n}, \zeta_{n}\right)_{n \geq 0} \in \mathcal{D}_{i_{0}}$. Since $\tau_{1}^{*}$ is optimal, we deduce from parts (i) and (ii) of Proposition 4.1 
that

$$
\begin{aligned}
Y_{0}^{0, i_{0}, X_{0}} & \geq \mathbf{E}\left[\int_{0}^{\tau_{1}} \psi_{i_{0}}\left(s, X_{s}^{0, i_{0}, X_{0}}\right) d s+\mathcal{O}_{\tau_{1}}^{0, i_{0}, x_{0}} \mathbf{1}_{\tau_{1}<T}+g_{i_{0}}\left(X_{T}^{0, i_{0}, X_{0}}\right) \mathbf{1}_{\tau_{1}=T}\right] \\
& \geq \mathbf{E}\left[\int_{0}^{\tau_{1}} \psi_{\zeta_{0}}\left(s, X_{s}^{\alpha}\right) d s+\left(Y_{\tau_{1}}^{\tau_{1}, \zeta_{1}, X_{\tau_{1}}^{\alpha}}-c_{\zeta_{0}, \zeta_{1}}\left(\tau_{1}\right)\right) \mathbf{1}_{\tau_{1}<T}+g_{i_{0}}\left(X_{T}^{\alpha}\right) \mathbf{1}_{\tau_{1}=T}\right] .
\end{aligned}
$$

Proceeding exactly as in step 1, an induction argument leads to

$$
\begin{aligned}
Y_{0}^{0, i_{0}, X_{0} \geq \mathbf{E}\left[\int_{0}^{\tau_{n}} \psi_{\alpha_{s}}\left(X_{s}^{\alpha}\right) d s+Y_{\tau_{n}}^{\tau_{n}, \zeta_{n}, X_{\tau_{n}}^{\alpha}} \mathbf{1}_{\tau_{n}<T}\right.} \\
\left.\quad-\sum_{k=1}^{n} c_{\zeta_{k-1}, \zeta_{k}}\left(\tau_{k}\right) \mathbf{1}_{\tau_{k}<T}+\sum_{k=1}^{n} g_{\zeta_{k-1}^{*}}\left(X_{T}^{\alpha}\right) \mathbf{1}_{\tau_{k-1}^{*}<\tau_{k}^{*}=T}\right], \quad n \in \mathbb{N}^{*} .
\end{aligned}
$$

Sending $n$ to infinity, since the strategy $\alpha$ is finite and (H2)(iii) is satisfied, we get

$$
Y_{0}^{0, i_{0}, X_{0}} \geq \mathbf{E}\left[\int_{0}^{T} \psi_{\alpha_{s}}\left(X_{s}^{\alpha}\right) d s-\sum_{k \geq 1} c_{\zeta_{k-1}, \zeta_{k}}\left(\tau_{k}\right) \mathbf{1}_{\tau_{k}<T}+g_{\alpha_{T}}\left(X_{T}^{\alpha}\right)\right]=J(\alpha) .
$$

The arbitrariness of $\alpha \in \mathcal{D}_{i_{0}}$ concludes the proof.

\subsection{Alternative introduction of constrained jumps}

This last paragraph is devoted to the proof of Theorem 3.3 . Using a penalization argument, we verify the existence of a minimal solution to the one-dimensional constrained BSDE with jumps (3.10)- $(3.9)$ and relate it to the members of the BSDE family (4.1). Using Proposition 4.2 , this leads directly to the the optimal switching interpretation of the solution.

Proof of Theorem 3.3. The proof is performed in four steps. We first verify that 3.9 admits a unique minimal solution. Then, we introduce a sequence of penalized BSDEs based on the family of reflected BSDEs (4.1). We finally verify that this sequence of BSDEs converges indeed to the minimal solution of $(3.9)$, which leads to the announced alternative BSDE representation for $J_{I_{0}}^{*}$.

Step 1. Existence of a unique minimal solution to (3.9).

Consider the triplet $\left(\bar{Y}^{\prime}, \bar{Z}^{\prime}, \bar{U}^{\prime}\right)$ defined by

$$
\bar{Y}_{t}^{\prime}:=Y_{t}^{t, I_{t}, X_{t}^{I}}, \quad \bar{Z}_{t}^{\prime}:=Z_{t}^{t, I_{t^{-}}, X_{t}^{I}} \quad \text { and } \quad \bar{U}_{t}^{\prime}(i):=Y_{t}^{t, i, X_{t}^{I}}-Y_{t}^{t, I_{t^{-}}, X_{t}^{I}}, \quad t \in[0, T] .
$$

Using Proposition 4.1(i), we obtain that

$$
\bar{Y}_{t}^{\prime}=\sum_{i \geq 0} \mathbf{1}_{\left[\tau_{i}, \tau_{i+1}\right)}(t) Y_{t}^{\tau_{i}, \zeta_{i}, X_{\tau_{i}}^{I}}, \quad t \in[0, T],
$$

where $\left(\tau_{0}, \zeta_{0}\right)=\left(0, I_{0}\right)$ and we recall that $\mu=\sum_{i \geq 1} \delta_{\left(\tau_{i}, \zeta_{i}\right)}$, i.e. $\left(\tau_{i}\right)_{i \geq 1}$ and $\left(\zeta_{i}\right)_{i \geq 1}$ correspond respectively to the jump times and marks of the random measure $\mu$. Then, a direct 
computation shows that $\left(\bar{Y}^{\prime}, \bar{Z}^{\prime}, \bar{U}^{\prime}, \bar{K}^{\prime}\right)$ solves 3.9$)$ where $\bar{K}^{\prime}$ is a process in $\mathbf{A}_{\mathbb{G}}^{2}$ defined by

$$
\bar{K}_{t}^{\prime}=\sum_{i \geq 0}\left(K_{t \wedge \tau_{i+1}}^{\tau_{i}, \zeta_{i}, X_{\tau_{i}}^{I}}-K_{t \wedge \tau_{i}}^{\tau_{i}, \zeta_{i}, X_{\tau_{i}}^{I}}\right), \quad t \in[0, T] .
$$

Hence, under (H2), we can apply Theorem 2.1 in [9] and we get the existence of a unique minimal solution $(\bar{Y}, \bar{Z}, \bar{U}, \bar{K})$ to 3.9 .

Step 2. Introduction of a sequence of penalized BSDEs associated to the BSDEs with oblique reflections.

For any $(\nu, \eta) \in \mathcal{K}$ and $n \in \mathbb{N}$, let define the sequence of processes $\left(Y^{\nu, i, \eta, n}, Z^{\nu, i, \eta, n}, K^{\nu, i, \eta, n}\right)_{i \in \mathcal{I}} \in$ $\left(\mathcal{S}_{\mathbb{F}}^{2} \times \mathbf{L}_{\mathbb{F}}^{2}(\mathbf{W}) \times \mathbf{A}_{\mathbb{F}}^{\mathbf{2}}\right)^{\mathcal{I}}$ as the solution of the penalized BSDE

$$
\begin{aligned}
Y_{t}^{\nu, i, \eta, n}= & g_{i}\left(X_{T}^{\nu, i, \eta}\right)+\int_{t}^{T} \psi_{i}\left(s, X_{s}^{\nu, i, \eta}\right) d s-\int_{t}^{T}\left\langle Z_{s}^{\nu, i, \eta, n}, d W s\right\rangle \\
& +n \int_{t}^{T}\left\{\sum_{j \in \mathcal{I}}\left[Y_{s}^{\nu, j, \eta, n}-c_{i, j}(s)-Y_{s}^{\nu, i, \eta, n}\right]^{-} \lambda(j)\right\} d s, \quad i \in \mathcal{I}, \quad 0 \leq t \leq T .
\end{aligned}
$$

Since this BSDE is Lipschitz, it admits a unique solution in $\left(\mathcal{S}_{\mathbb{F}}^{2} \times \mathbf{L}_{\mathbb{F}}^{2}(\mathbf{W}) \times \mathbf{A}_{\mathbb{F}}^{2}\right)^{\mathcal{I}}$. According to Theorem 2.1 in [12], the sequence $\left(Y^{\nu, ., \eta, n}\right)_{n \in \mathbb{N}}$ is nondecreasing and converges in $\mathcal{S}_{\mathbb{F}}^{2}$ to $\left(Y^{\nu, i, \eta}\right)_{i \in \mathcal{I}}$ as $n$ goes to $\infty$, for each $(\nu, \eta) \in \mathcal{K}$. In particular the sequence $\left(Y_{0}^{0, I_{0}, X_{0}, n}\right)_{n \geq 0}$ converges to $\left(Y_{0}^{0, I_{0}, X_{0}}\right)=J_{I_{0}}^{*}$.

Step 3. Link with penalized BSDEs associated to the constrained BSDE.

For any $n \geq 0$, we introduce the triplet $\left(\bar{Y}^{n}, \bar{Z}^{n}, \bar{U}^{n}\right)$ given by

$$
\bar{Y}_{t}^{n}:=Y_{t}^{t, I_{t}, X_{t}^{I}, n}, \quad \bar{Z}_{t}^{n}:=Z_{t}^{t, I_{t^{-}}, X_{t}^{I}, n} \quad \text { and } \quad \bar{U}_{t}^{n}(i):=Y_{t}^{t, i, X_{t}^{I}, n}-Y_{t^{-}}^{t, I_{t^{-}}, X_{t}^{I}, n}, \quad t \leq T,
$$

where we recall that $\left(I, X^{I}\right)$ is defined by 3.10 . A direct computation shows that $\left(\bar{Y}^{n}, \bar{Z}^{n}, \bar{U}^{n}\right)$ solves the following BSDE with jumps

$$
\begin{aligned}
\bar{Y}_{t}^{n}=g_{I_{T}}\left(X_{T}^{I}\right) & +\int_{t}^{T} \psi_{I_{s}}\left(s, X_{s}^{I}\right) d s+n \int_{t}^{T} \int_{\mathcal{I}^{\prime}}\left[\bar{U}_{s}^{n}(i)+c_{I_{s^{-}}, i}(s)\right]^{-} \lambda(d i) d s \\
& -\int_{t}^{T}\left\langle\bar{Z}_{s}^{n}, d W_{s}\right\rangle-\int_{t}^{T} \int_{\mathcal{I}} \bar{U}_{s}^{n}(i) \mu(d s, d i), \quad 0 \leq t \leq T
\end{aligned}
$$

for any $n \geq 0$.

Step 4. Convergence of the sequence of penalized solutions

Observe that the sequence of BSDEs 4.15 satisfied by $\left(\bar{Y}^{n}, \bar{Z}^{n}, \bar{U}^{n}\right)_{n}$ corresponds exactly to a penalized version of the constrained BSDE with jump of interest (3.9). From Step 1 and Theorem 2.1 in [9], the sequence $\left(\bar{Y}^{n}\right)_{n}$ converges increasingly to $\bar{Y}$, the first component of the minimal solution to 3.9 . Letting $n$ go to infinity in the relation $\bar{Y}_{0}^{n}=Y_{0}^{0, I_{0}, X_{0}, n}$, we deduce from Step 2 and 4.12 that $\bar{Y}_{0}=Y_{0}^{0, I_{0}, X_{0}}=J_{I_{0}}^{*}$. 


\section{References}

[1] B. Bouchard, A stochastic target formulation for optimal switching problems in finite horizon, Stochastics 81 (2) (2009) 171-197.

[2] R. Carmona and M. Ludkovski, Pricing Asset Scheduling Flexibility Using Optimal Switching, Applied Mathematical Finance 15 (6) (2008) 405-447.

[3] J.-F. Chassagneux, R. Elie and I. Kharroubi, A note on existence and uniqueness for solutions of multidimensional reflected BSDEs, Electronic Communications in Probability 16 (2011) 120-128.

[4] J.-F. Chassagneux, R. Elie and I. Kharroubi, Discrete-time Approximation of Multidimensional BSDEs with oblique reflections, Annals of Applied Probability 22 (3) (2012) 971-1007.

[5] B. Djehiche, S. Hamadène and I. Hdhiri, Stochastic Impulse Control for Non-Markovian Processes, Applied Mathematics and Optimization 61 (2010) 1-26.

[6] B. Djehiche, S. Hamadène and A. Popier, The finite horizon optimal multiple switching problem, SIAM Journal on Control and Optimization 48 (4) (2009) 2751-2770.

[7] N. El Karoui, C. Kapoudjian, E. Pardoux, S. Peng and M.C. Quenez, Reflected solutions of Backward SDE's, and related obstacle problems for PDEs, Annals of Probability 25 (1997) 702-737.

[8] R. Elie and I. Kharroubi, Probabilistic representation and approximation for coupled systems of variational inequalities, Statistics and Probability Letters 80 (17-18) (2009) 1388-1396.

[9] R. Elie and I. Kharroubi, Adding constraints to BSDEs with Jumps: an alternative to multidimensional reflections, forthcoming in ESAIM: Probability and Statistics (2010). DOI: $10.1051 / \mathrm{ps} / 2013036$.

[10] S. Hamadène and M. Jeanblanc, On the starting and stopping problem: application in reversible investments. Mathematics of Operations Research 32 (2007) 182-192.

[11] S. Hamadène and J. Zhang, A Switching Problem and Related System of Reflected Backward SDEs. Stochastic Processes and Applications 120 (2010) 403-426.

[12] Y. Hu and S. Tang, Multi-dimensional BSDE with oblique Reflection and optimal switching, Probability Theory and Related Fields 147(1-2) (2010) 89-121.

[13] A. Porchet, N. Touzi and X. Warin, Valuation of power plants by utility indifference and numerical computation, Mathematical Methods of Operations Research 70 (1) (2008) 47-75. 